\title{
An unrestrained proinflammatory M1 macrophage population induced by iron impairs wound healing in humans and mice
}

\author{
Anca Sindrilaru, ${ }^{1}$ Thorsten Peters, ${ }^{1}$ Stefan Wieschalka, ${ }^{1}$ Corina Baican, ${ }^{2}$ Adrian Baican, ${ }^{2}$ \\ Henriette Peter, ${ }^{1}$ Adelheid Hainzl, ${ }^{1}$ Susanne Schatz, ${ }^{1}$ Yu Qi, ${ }^{1}$ Andrea Schlecht, ${ }^{1}$ Johannes M. Weiss, ${ }^{1}$ \\ Meinhard Wlaschek, ${ }^{1}$ Cord Sunderkötter, ${ }^{3}$ and Karin Scharffetter-Kochanek ${ }^{1}$ \\ 1Department of Dermatology and Allergic Diseases, University of UIm, Ulm, Germany. 'Department of Dermatology and Venereology, \\ University of Cluj-Napoca, Cluj-Napoca, Romania. ${ }^{3}$ Department of Dermatology, University of Münster, Münster, Germany.
}

\begin{abstract}
Uncontrolled macrophage activation is now considered to be a critical event in the pathogenesis of chronic inflammatory diseases such as atherosclerosis, multiple sclerosis, and chronic venous leg ulcers. However, it is still unclear which environmental cues induce persistent activation of macrophages in vivo and how macrophage-derived effector molecules maintain chronic inflammation and affect resident fibroblasts essential for tissue homeostasis and repair. We used a complementary approach studying human subjects with chronic venous leg ulcers, a model disease for macrophage-driven chronic inflammation, while establishing a mouse model closely reflecting its pathogenesis. Here, we have shown that iron overloading of macrophages - as was found to occur in human chronic venous leg ulcers and the mouse model - induced a macrophage population in situ with an unrestrained proinflammatory M1 activation state. Via enhanced TNF- $\alpha$ and hydroxyl radical release, this macrophage population perpetuated inflammation and induced a $16^{\mathrm{INK}}{ }^{\mathrm{Na}}$-dependent senescence program in resident fibroblasts, eventually leading to impaired wound healing. This study provides insight into the role of what we believe to be a previously undescribed iron-induced macrophage population in vivo. Targeting this population may hold promise for the development of novel therapies for chronic inflammatory diseases such as chronic venous leg ulcers.
\end{abstract}

\section{Introduction}

Chronic venous leg ulcers (CVUs) represent the final outcome of lower-extremity chronic venous insufficiency in most cases $(1,2)$. It is a debilitating, recurrent complication with a steady increase in incidence with age (3).

CVUs fail to progress through the normal pattern of wound repair, involving inflammation, granulation tissue formation, and remodeling (4), but instead remain in a chronic inflammatory state with little signs of healing (1, 2, 5-9). Accumulation of activated macrophages, as indicated by NOS expression, is a key feature in CVUs $(10,11)$. In addition, neutralization of TNF- $\alpha$, a cytokine released by various cell types, including macrophages, has substantially accelerated healing of CVUs in a series of 14 nonhealing ulcers (12). However, to our knowledge, neither the role of macrophages in perpetuating chronic inflammation nor the microenvironmental cues in CVUs that may lead to persistent monocyte/macrophage activation have been addressed in sufficient detail. So far, there is evidence that venous hypertension - caused by venous valve incompetence - results in venous stasis of the lower extremity, low or absent shear stress, and hypoxia, which all trigger endothelial cell activation with "trapping" of monocytes/macrophages and other leukocytes in the microcirculation (13-15). Activated monocytes/macrophages and other leukocytes transmigrate into the tissue and release high amounts of proinflammatory cytokines (16), proteases $(17)$, and $\operatorname{ROS}(18,19)$, which are responsible for the breakdown of the connective tissue and of essential growth factors (20-22), eventually leading to ulcer formation $(1,2,9)$.

Conflict of interest: The authors have declared that no conflict of interest exists. Citation for this article: J Clin Invest. 2011;121(3):985-997. doi:10.1172/JCI44490.
Although not systematically studied, macrophages in CVUs have been reported to contain high amounts of Prussian blue-positive iron $(23,24)$, most likely due to extravasation of erythrocytes secondary to venous hypertension and stasis. Erythrocytes are engulfed by macrophages, and the released iron is bound to intracellular ferritin, which over time changes its structure to hemosiderin (23, 24). This contributes to an up to 20 -fold higher iron concentration in the lower limbs of CVU patients than in the upper arms of the same patients (25). Iron overload in tissue and wound exudates of CVUs, but not of acute wounds (AWs), was confirmed in 2 independent studies $(18,19)$. In the presence of $\mathrm{H}_{2} \mathrm{O}_{2}$ released by macrophages and neutrophils, iron drives the generation of highly toxic hydroxyl radicals $\left(\mathrm{OH}^{*}\right)$ via the Fenton reaction. In fact, significant oxidative damage was found in CVUs $(18,19)$. The pathogenic role of iron-overloaded macrophages in the perpetuated inflammatory nonhealing state of CVUs is poorly understood. In contrast, the requirement of macrophages for physiologic tissue repair in adults is better understood and is supported by early depletion experiments using antimacrophage serum (26) and, more recently, by murine knockout models. These models revealed impaired wound healing caused by reduced numbers or functional defects of macrophages at distinct phases of tissue repair $(20,27-30)$. During physiologic wound healing, macrophages ingest and combat invading microbes, contribute to debris scavenging, and enhance tissue remodeling by the release of a wide variety of growth factors.

Tissue macrophages originate from peripheral blood monocytes and reveal a substantial heterogeneity of phenotypes and specialization (31). In analogy to the concept of the Th1/Th2 dichotomy, macrophages have been classified as M1 as opposed to M2 based on in vitro data (31-34). According to this classification, the M1 designation was reserved for classically activated macrophages fol- 
lowing stimulation with IFN- $\gamma$ and LPS (which induces TNF- $\alpha$ ), and the M2 designation was applied to the alternatively activated macrophages after in vitro stimulation with IL-4 and IL-13 (35). M1 macrophages reveal enhanced microbicidal capacity and secrete high levels of proinflammatory cytokines (TNF- $\alpha$, IL-1, IL-6, and IL-23) and increased concentrations of superoxide anions $\left(\mathrm{O}_{2}^{-\bullet}\right)$, oxygen radicals, and nitrogen radicals to increase their killing activity, as required in early phases of tissue repair (36). Conversely, M2 macrophages dampen proinflammatory cytokine levels, secrete components of the extracellular matrix, and may be essential for late phases of tissue repair. Even though the validity of the M1/M2 paradigm in vivo is largely unexplored, recent analysis of the dynamics of macrophage-specific gene expression during physiologic wound healing showed that wound macrophages display a similar M1 gene expression profile in early healing phases, which is later changed in favor of antiinflammatory M2 macrophage-related genes. Beyond the correlative nature of increased iron deposits in macrophages and disease progression in CVUs, it is unknown whether and how iron affects the M1/M2 dichotomy and activation state of macrophages. This is of substantial clinical relevance, as it may lead to the identification of early steps in the pathogenesis of CVUs. We therefore set out to study the causal role of iron in the induction and perpetuation of the activation state of macrophages and effector molecules leading to persistent inflammation, tissue breakdown, and impaired tissue restoration.

Using iron chelation, depletion of macrophages, and the TNF- $\alpha$ antagonist etanercept, we here describe an iron-induced macrophage population in CVUs and the corresponding murine model, which we believe to be novel, with an unrestrained proinflammatory M1 activation state along with the highly expressed M2 iron scavenger receptor CD163. These macrophages, via the release of high concentrations of TNF- $\alpha$ and $\mathrm{OH}^{*}$, are responsible for the perpetuation of inflammation and premature aging of resident ulcer-adjacent fibroblasts no longer available for productive tissue repair. The identification of these molecular and cellular key players in CVUs may contribute to the development of novel therapies targeting this distinct macrophage population or iron also in other chronic inflammatory diseases.

\section{Results}

Activated macrophages accumulate and persist in CVUs. Normal wound healing follows a sequence of events involving clotting, inflammation, matrix deposition, and remodeling $(4,37)$. Within a few hours after injury, first polymorphonuclear neutrophils and later macrophages invade the wound site. Both neutrophils and macrophages produce proteinases and ROS to combat contaminating microorganisms and phagocytose cellular debris. In CVUs, this inflammatory phase is persistent $(9,10)$. Whether macrophages play a role in maintaining this persistent inflammation is not known.

Biopsies derived from CVU patients presented with high numbers of $\mathrm{CD} 68^{+}$macrophages, representing up to $80 \%$ of the cells in the wound margins (Figure 1A and Supplemental Figure 1; supplemental material available online with this article; doi:10.1172/ JCI44490DS1). These macrophages showed an increase in classical proinflammatory M1 markers iNOS and TNF- $\alpha$ in all studied biopsies derived from CVU patients (patients 1-5; Figure 1, B and $\mathrm{C}$, and Supplemental Table 1). In contrast, only a transient increase in macrophage numbers expressing both iNOS and TNF- $\alpha$ were observed in AWs at day 2 after wounding, with a subsequent reduction in macrophage numbers to those of normal skin (NS) by day 5 (Figure $1, \mathrm{~A}-\mathrm{C}$ ). iNOS generates microbicidal $\mathrm{NO}^{\bullet}$ to combat invading microbes. TNF- $\alpha$ stimulates iNOS synthesis and thus enhances microbicidal defense. However, persistent overproduction of TNF- $\alpha$, as observed here in CVUs, may be detrimental for tissue repair. In fact, injection of recombinant TNF- $\alpha$ (rTNF- $\alpha$ ) in the wound margins of AWs in a mouse model of full-thickness excisional wounds significantly delayed wound healing (Supplemental Figure 2).

Macrophages mount a distinct activation phenotype in CVUs. We hypothesized that macrophages from CVUs fail to switch from proinflammatory M1 macrophages to the antiinflammatory M2 macrophages required for tissue remodeling and restoration. We studied proinflammatory M1 activation markers, including cytokines (TNF- $\alpha$ and IL-12/IL-23), chemokine receptors involved in monocyte recruitment and activation (CCR2 and $\mathrm{CX}_{3} \mathrm{CR} 1$ ), and iNOS, which is involved in phagocytosis and bacterial killing (32, 38). In addition, we included antiinflammatory M2 markers like arginase, which exerts its antiinflammatory effect via degradation of arginine required for iNOS activity, and M2 nonopsonic scavenger (CD36), $\beta$-glucan (Dectin-1), and mannose receptors (CD206) $(35,39)$. We performed double immunostaining for combinations of M1 and M2 activation markers in AWs compared with CVUs using immunohistology and flow cytometric analysis of macrophages from enzymatically digested tissue samples (Figure 2, A-C). In selected experiments, macrophages gated on $\mathrm{CD}^{+} 8^{+}$were subsequently regated for M1 and M2 markers in order to demonstrate that M1 and M2 markers are expressed by the same macrophages. An unexpected transient concomitant expression of both proinflammatory M1 (TNF- $\alpha$, IL-12, CCR2, and CX ${ }_{3}$ CR1) and antiinflammatory M2 markers (CD206, arginase, Dectin-1, IL-10, IL-4R $\alpha$, CD36, and CD163) by almost all macrophages was observed in early phases of AWs at day 2 (Figure 2 and data not shown). Virtually all proinflammatory M1 markers were downregulated to basal levels in NS macrophages, and high expression of antiinflammatory M2 markers prevailed in macrophages of AWs at day 5 after wounding (Figure 2). Conversely, persistent coexpression of high levels of proinflammatory M1 markers (TNF- $\alpha$, IL-12p40, and CCR2) and - apart from high expression of CD163 - intermediate expression levels of M2 markers (arginase, CD206, Dectin-1, IL-10, IL-4R $\alpha$, and CD36) was observed in all macrophages in the studied tissue samples from CVU patients (Figure 2, B and C).

These data suggested that a previously undescribed macrophage population in CVUs fails to fully switch from a proinflammatory M1 state to an antiinflammatory M2 program to efficiently promote angiogenesis, connective tissue deposition, and wound repair. These macrophages, with an unrestrained proinflammatory M1 activation state along with highly expressed M2 iron scavenger receptor CD163, are referred to herein as macrophages with unrestrained proinflammatory M1 phenotype.

Unrestrained proinflammatory $M 1$ macrophages accumulate iron in CVUs. Iron deposits in CVUs cause visible brownish skin that always surrounds chronic leg ulcers. The origin of increased iron stores in the skin around CVUs is extravasation of red blood cells in conditions of significantly increased blood pressure and venous stasis due to venous valve insufficiency. The observed enhanced expression of the hemoglobin-haptoglobin scavenger receptor CD163, an important M2 marker, is a consequence of red blood cell extravasation and precedes the engulfment and degradation by tissue macrophages (40). In addition, CD163 expressed on macrophages serves as scavenger receptor for hemoglobin-haptoglobin-bound iron, further increas- 
A

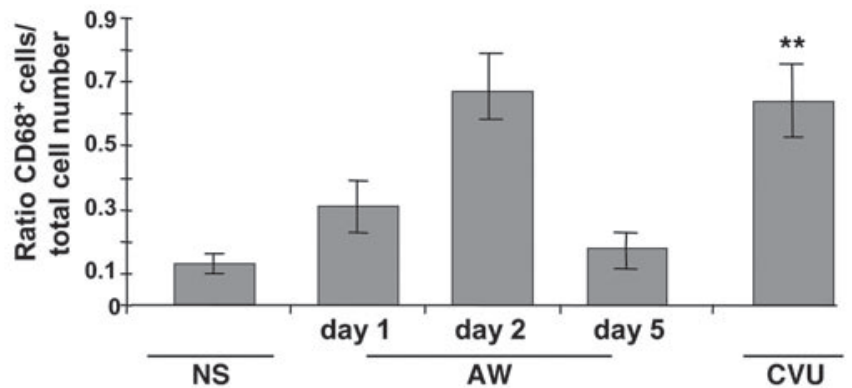

B

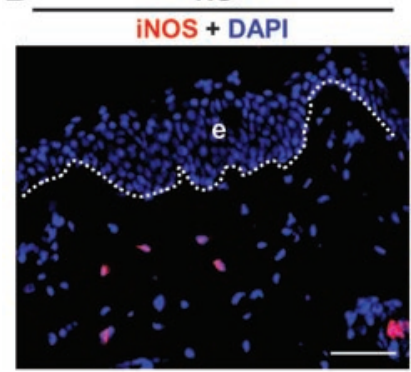

NS

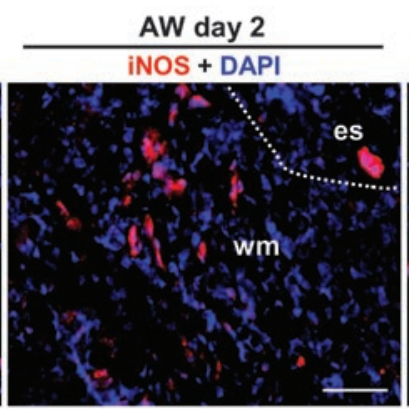

C

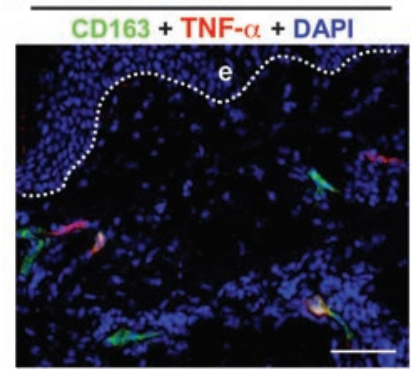

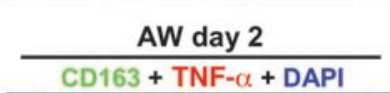

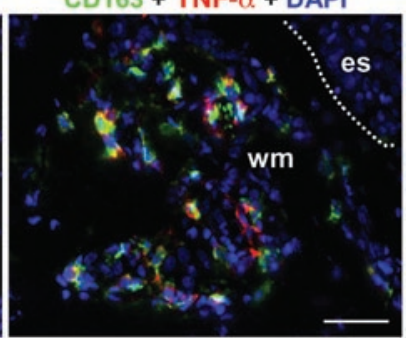

CVU
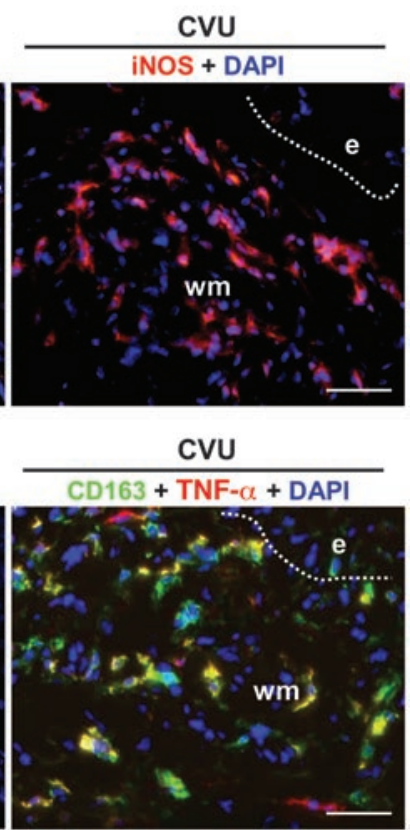

\section{Figure 1}

Activated macrophages accumulate and persist in CVUs. (A) Quantification of CD68+ infiltrating macrophages in NS, AWs at days 1, 2, and 5 after wounding, and CVUs were counted in 10 high-power fields per sample. Results are mean \pm SD ratio of $\mathrm{CD}^{+} 8^{+}$to total cells counted in the dermis $(n=5)$. ${ }^{*} P<0.01$ versus day- 5 AW and NS. (B and C) Representative photomicrographs of skin sections. Activation of macrophages was assessed in NS, AWs, and CVUs by immunostaining of cryosections for the classical activation markers (B) iNOS and (C) TNF- $\alpha$ or the nonclassical activation marker CD163. Nuclei were counterstained with DAPI. Scale bars: $100 \mu \mathrm{m}$. Dashed lines indicate the junction between epidermis (e) and dermis. es, eschar; wm, wound margin. ing intracellular iron stores. Consistent with previous data on tissue iron accumulation in CVUs $(18,19,25)$, we found accumulation of ferric iron in macrophages and in the extracellular space in all studied CVU samples (patients 1-5 and 12-16; Figure 2D and Supplemental Table 1). Conversely, iron deposits were absent in AWs. These data indicate that the $\mathrm{CD}_{163}{ }^{+}$macrophages with unrestrained proinflammatory M1 activation colocalize with iron in CVUs.

Iron causes the induction of the unrestrained proinflammatory $M 1$ macrophage population. In order to assess whether iron can induce the unrestrained proinflammatory activation of the newly defined macrophage population, we established a murine model closely reflecting main pathogenic aspects of CVUs. Repeated treatment of mice with iron-dextran resulted in an abundant deposition of iron in cells within the dermis of the skin (Figure 3A). After wounding, iron accumulated inside the $\mathrm{F} 4 / 80^{+}$macrophages. Iron loading of macrophages was almost completely prevented when treatment with iron-dextran was followed by injection of the iron chelator desferrioxamine (DFX; Figure 3A). Similar to human macrophages in CVUs, murine macrophages concomitantly expressed both M1 (TNF- $\alpha$ and IL-12) and M2 (CD206 and arginase) activation markers in wound margins from iron-dextran-treated mice in the early (day 2) and late (day 5) inflammatory phases of wound healing (Supplemental Figure 3A). Notably, macrophages isolated from wound margins of iron-dextran-treated mice revealed an activation pattern reminiscent of macrophages isolated from CVUs with a persistent proinflammatory M1 response and intermediate antiinflammatory M2 marker activation (i.e., TNF- $\alpha^{\text {hi }}$, IL-12 ${ }^{\text {hi }}$, CCR2 ${ }^{\text {hi }}$, Ly 6 Chi, Dectin- $1^{\text {med }}$, IL-4R $\alpha^{\text {med }}$, and CD204 ${ }^{\mathrm{med}}$ ) at day 5 after wounding compared with PBS-dextran-treated control mice (Figure 3B and Supplemental Figure 3B). Even though the nonopsonic scavenger receptors CD206 and CD301 were expressed at comparable levels in iron-dextran- and PBS-dextran-treated control mice and the M2 marker CD163 was highly expressed, the overall antiinflammatory $\mathrm{M} 2$ response may not be sufficient to terminate inflammation and promote tissue repair (Figure 3B). Consistent with the phenotype observed in vivo in CVUs and in the murine model, human macrophages mounted an identical activation profile, with dominance of proinflammatory markers when cultured under Fenton reactionmimicking conditions in the presence of Fe(III) chloride/ascorbate and $\mathrm{H}_{2} \mathrm{O}_{2}$ in vitro (Supplemental Figure 4).

TNF- $\alpha$ from iron-induced unrestrained proinflammatory M1 activated macrophages is responsible for impaired wound healing. Compared with PBS-dextran-treated mice, iron-loaded mice showed delayed wound healing at days 3, 5, 7, and 10 after wounding (Supplemental Figure 5A). Interestingly, whereas in PBS-treated wounds, macrophages reached a maximal number at day 2 after wounding and continuously decreased thereafter, macrophages accumulated and persisted at significantly higher numbers in iron-overloaded 
A

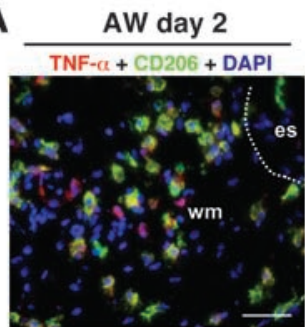

IL-12 + Arginase + DAPI
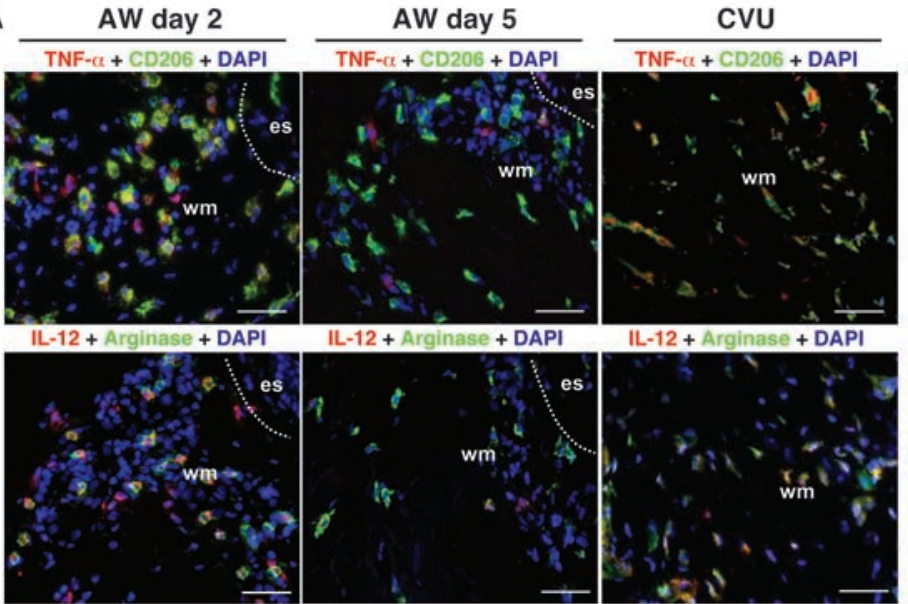

IL-12 + Arginase + DAPI

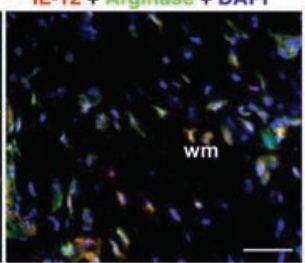

B
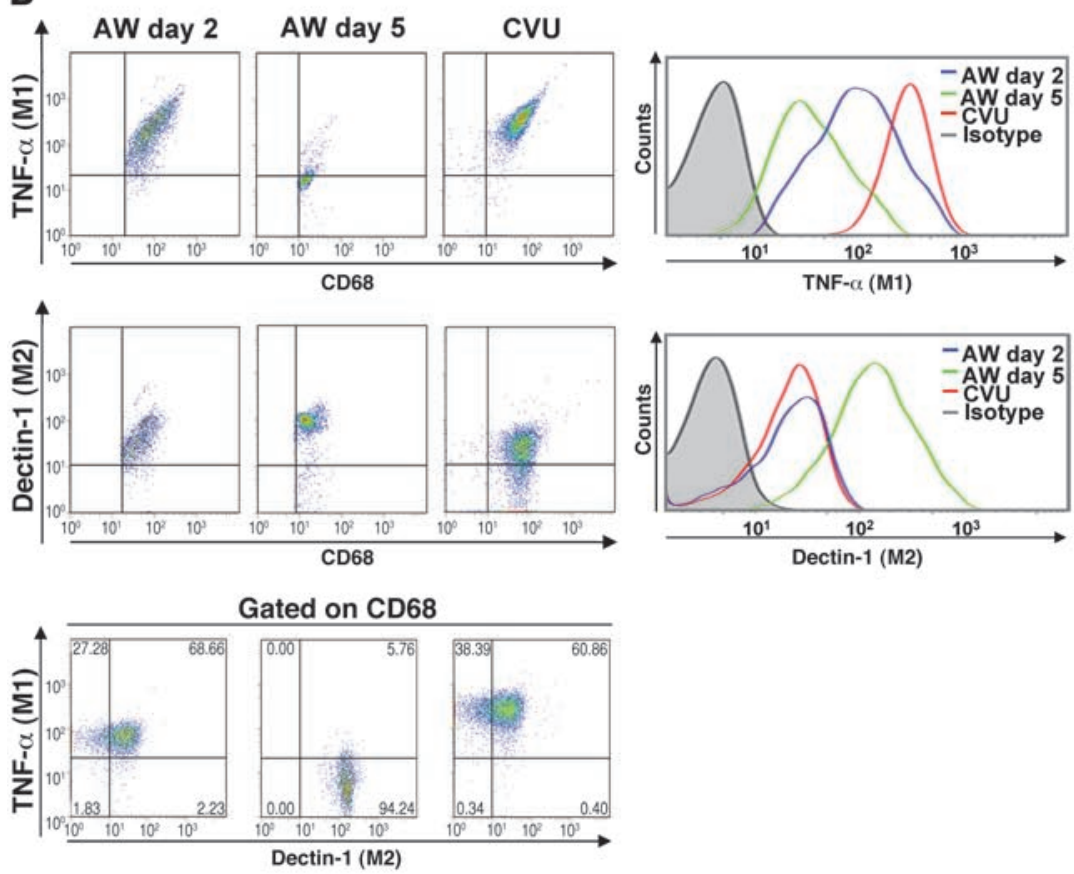

\section{D}
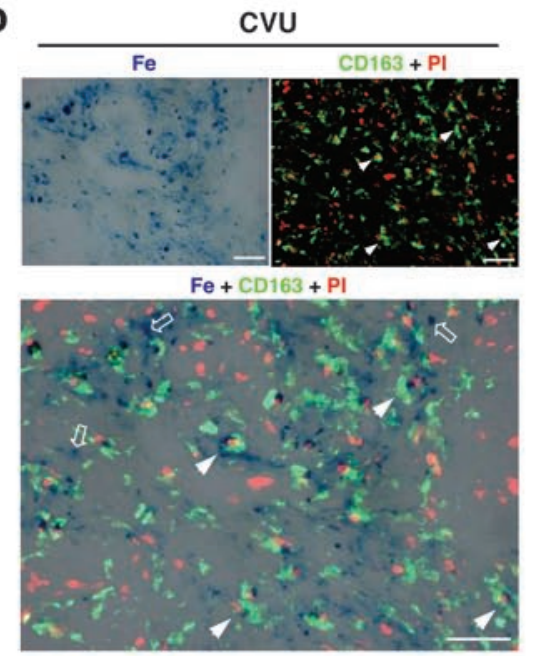

AW day 2

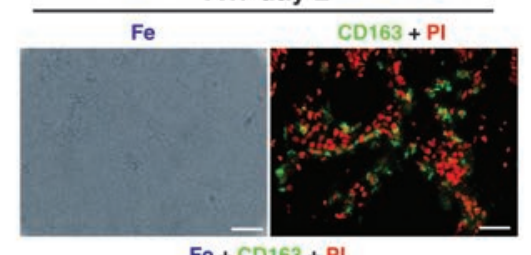

$\mathrm{Fe}+\mathrm{CD} 163+\mathrm{PI}$

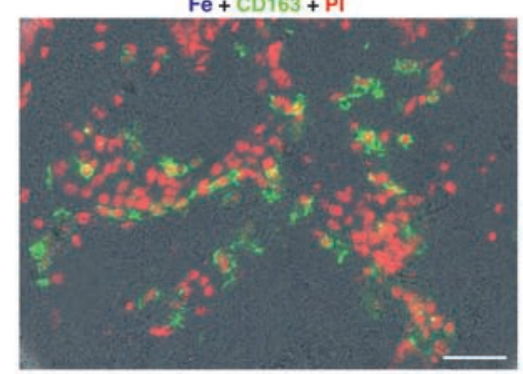

C

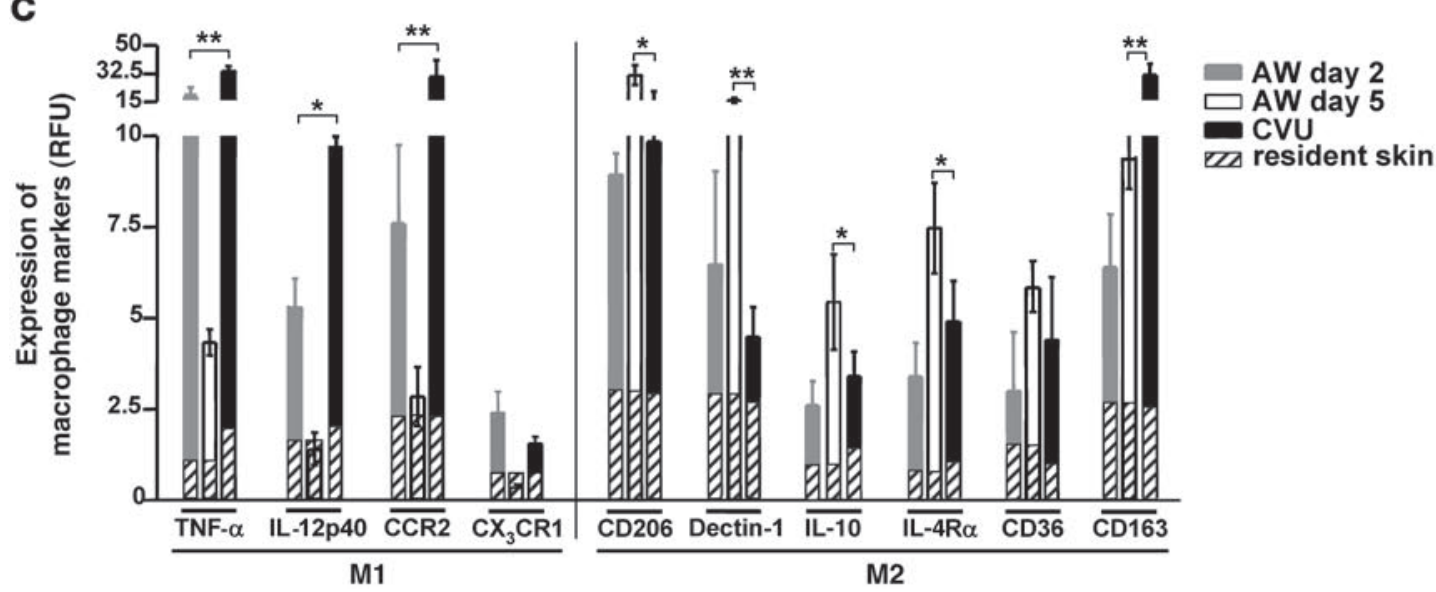




\section{Figure 2}

The identified macrophage population mounts an unrestrained proinflammatory M1 activation phenotype and accumulates iron in CVUs. (A) Representative photomicrographs with double immunostaining of skin cryosections from AWs and CVUs for M1 and M2 macrophage activation markers TNF- $\alpha$ and CD206 or IL-12 and arginase-1. Nuclei were stained with DAPI. Scale bars: $100 \mu \mathrm{m}$. Dashed lines indicate the junction between eschar and wound margin. (B) Flow cytometry analysis of wound macrophages purified from AW tissue 2 and 5 days after wounding and CVUs gated according to side scatter (SSC) and CD68 and regated for CD68 and M1 marker TNF- $\alpha$, CD68 and M2 marker Dectin-1, or TNF- $\alpha$ and Dectin-1. (C) Expression levels for M1 and M2 activation markers of macrophages isolated from $5 \mathrm{AWs}$ and $6 \mathrm{CVUs}$ by flow cytometry. Results are given in RFU (see Methods). Resident skin macrophages were pooled from NS $(n \geq 5)$. ${ }^{*} P<0.05$, ${ }^{* *} P<0.01$, Student's $t$ test. (D) Representative photomicrographs of cryosections from CVU patients and AWs from healthy volunteers stained for iron by Perl Prussian blue and immunostained with CD163 for macrophages. Nuclei were stained with PI. High amounts of iron were identified within the $\mathrm{CD}_{163^{+}}$macrophages (filled arrows) and extracellular space (open arrows) in CVUs, but not AWs. Scale bars: $150 \mu \mathrm{m}$.

mice compared with control mice at days 3, 4, and 5 after wounding. These data suggest that increased numbers of activated, TNF- $\alpha$-producing macrophages are most likely responsible for the observed healing defect (Supplemental Figure 5, A and B).

The wound healing phenotype in iron-loaded mice was fully rescued in the presence of the iron chelator DFX (Figure 3, C and D), demonstrating the causal role of iron in delayed wound healing. TNF- $\alpha$ has previously been shown to maintain the activation state of macrophages in an autocrine manner $(41,42)$. This is in line with our observation that $\mathrm{F} 4 / 80^{+}$macrophages with unrestrained proinflammatory M1 phenotype highly expressed TNF- $\alpha$ in ironloaded compared with control mice, and that intracellular TNF- $\alpha$ was reduced in macrophages of iron-loaded mice in the presence of the TNF- $\alpha$ antagonist etanercept (Figure 3E). As TNF- $\alpha$ has previously been shown to maintain the activation state of macrophages in an autocrine manner, neutralization of soluble TNF- $\alpha$ by injection of etanercept most likely resulted in the disruption of this autocrine feedback loop and subsequently in reduced TNF- $\alpha$ synthesis by macrophages and dampened inflammation. Consistent with a causal role of TNF- $\alpha$ in delayed wound healing, etanercept injection at day 3 after wounding rescued impaired wound healing in iron-loaded mice at days 5, 7, and 10 after wounding (Figure 3, C and D), whereas injection of rTNF- $\alpha$ into the margins of AWs in control mice resulted in significant delay of wound healing (Supplemental Figure 2). Given that etanercept injection was performed at day 3 , a rescue of the delay in wound healing of day-3 wounds (Figure 3D) cannot be expected.

Virtually all F4/80+ macrophages of enzymatically digested wounds from iron-dextran-treated mice revealed the unrestrained proinflammatory M1 activation pattern. To determine whether the iron-activated proinflammatory TNF- $\alpha$-releasing macrophages are responsible for delayed wound healing, we depleted the macrophages from wound margins by injections of liposomes encapsulated by dichloromethylene diphosphonate (clodronate) at day 4 after wounding as previously described (41). As expected, 24 hours after injection of clodronate liposomes, macrophages were almost completely depleted (Supplemental Figure 6A), whereas numbers of mature dendritic cells, Langerhans cells, neutrophils, lymphocytes, and mast cells were not affected (Supplemental Figure 6,
B-G and refs. 41, 43). Injection of clodronate liposomes, but not control PBS liposomes, in the late inflammatory phase at day 4 after wounding resulted in a significant improvement of impaired wound healing of iron-loaded mice and fully rescued the delayed tissue repair at day 10 after wounding, whereas wound healing of PBS-dextran-treated mice injected with clodronate liposomes was not affected (Figure 3F). These data indicate that macrophages with unrestrained proinflammatory M1 phenotype are important in iron-induced delayed wound healing and imply that TNF- $\alpha$ release from M1 proinflammatory macrophages cause the irondependent impaired wound healing phenotype.

Iron-activated macrophages release toxic amounts of $\mathrm{OH}^{*}$ and peroxynitrite in vivo. Iron-loaded macrophages, while releasing $\mathrm{H}_{2} \mathrm{O}_{2}$, may drive the Fenton reaction and thus cause the generation of highly toxic $\mathrm{OH}^{\bullet}(9)$. Also, iron-activated macrophages release $\mathrm{O}_{2}^{-}$and $\mathrm{NO}^{*}$, which can generate peroxynitrite (ONOO$\left.{ }^{*}\right)$ (44). To determine whether production of noxious $\mathrm{OH}^{*}$ and ONOO ${ }^{\bullet}$ by ironactivated macrophages occurs in vivo, we applied the redox-sensitive dye dihydrorhodamine123 (DHR) on cryosections of NS, CVUs, and skin from PBS-dextran-treated and iron-loaded mouse wounds in the presence and absence of scavengers for distinct ROS. The wound margins of human AWs and CVUs presented with intense green fluorescence (Figure 4A), indicative of a strong oxidative burst. $\mathrm{O}_{2}{ }^{-}$and $\mathrm{H}_{2} \mathrm{O}_{2}$ were primarily responsible for the oxidative burst in AW, as coincubation of the sections with SOD, which detoxifies $\mathrm{O}_{2}^{-}$, and with the $\mathrm{H}_{2} \mathrm{O}_{2}$ scavenger catalase (Cat) clearly quenched the fluorescence intensity. DMSO, the scavenger for $\mathrm{OH}^{\bullet}$, did not quench the signal. Notably, $\mathrm{O}_{2}{ }^{-}$and $\mathrm{OH}^{\bullet}$, but not $\mathrm{H}_{2} \mathrm{O}_{2}$, were the prevailing ROS in all studied biopsies from CVU patients (Figure 4A). Identical results were found in wounds of iron-loaded mice compared with those of control mice (Supplemental Figure 7A). These differences in distinct ROS and concentrations may contribute to tissue damage and the nonhealing state of macrophage iron-overload conditions. In fact, immunostaining of sections with antibodies against 8-oxo-2'-deoxyguanosine (8OHdG), a marker for oxidative DNA damage, identified high numbers of cells with oxidative DNA damage in CVUs, but not in AWs or NS (Figure 4B). Similarly, an antibody against 3-nitrotyrosine (3-NT), a selective marker for protein nitration due to $\mathrm{ONOO}^{\circ}$, detected enhanced protein nitration in CVUs, but not in AWs or NS (Figure 4C). These data were confirmed in skin lysates from iron-loaded mice compared with PBS-treated mice and CVUs (Figure 4D and Supplemental Figure 8). Reduction of proinflammatory TNF- $\alpha$ released by macrophages with etanercept, or depletion of macrophages with clodronate liposomes, prevented generation of toxic $\mathrm{OH}^{*}$ and $\mathrm{ONOO}^{\circ}$ in iron-treated mice (Figure 4D and Supplemental Figure 7B). These data indicate a critical role for unrestrained proinflammatory M1 activated macrophages and enhanced TNF- $\alpha$ release for generation of toxic radicals.

Enhanced macrophage-dependent ROS release activates a senescence program. $\mathrm{OH}^{*}$ and $\mathrm{ONOO}^{*}$ can severely damage macromolecules and can induce double strand breaks and inter-crosslinks in the DNA (44). Such lesions induce a senescence program in resident skin fibroblasts $(45,46)$ and thus may contribute to impaired wound healing. To test this hypothesis, $\gamma \mathrm{H} 2 \mathrm{AX}$, a phosphorylated histone protein and aging marker (47) with specificity for DNA double strand breaks in vivo $(45,47)$, was studied. Double staining with antibodies against CD18, the common $\beta$ chain of $\beta_{2}$ integrins that is exclusively expressed on leukocytes, was used to differentiate $\gamma \mathrm{H} 2 \mathrm{AX}$ expression in inflammatory leukocytes from resident fibroblasts. In 

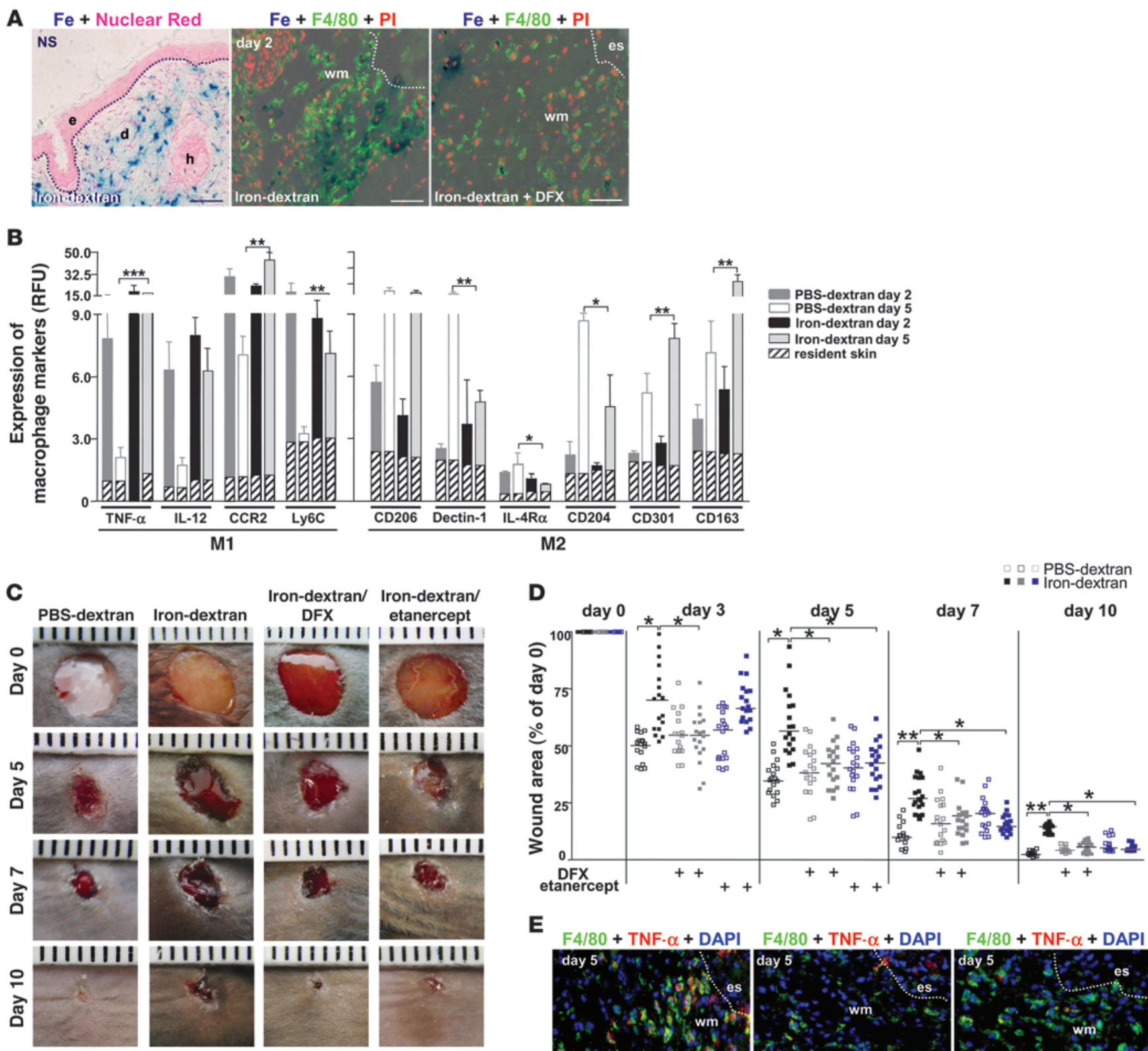

E

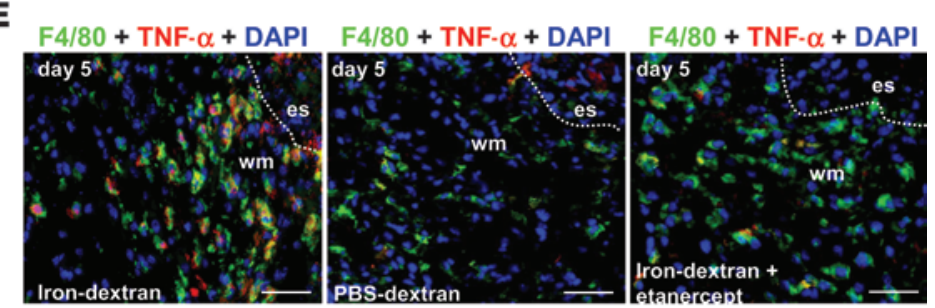

$\mathbf{F}$

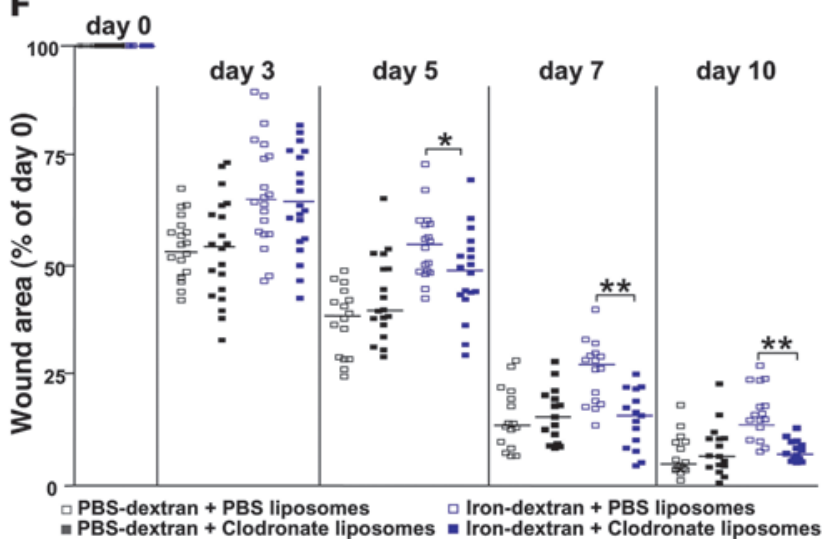




\section{Figure 3}

Iron is causal for induction of the macrophage population with unrestrained proinflammatory M1 phenotype and for impaired wound healing. (A) Representative photomicrographs detects Fe deposition in $\mathrm{NS}$ and in $\mathrm{F} 4 / 80^{+}$macrophages in iron-dextran-treated wounds. Iron deposition was almost completely prevented by coinjection with DFX. Cell nuclei were counterstained with PI. Scale bars: $150 \mu \mathrm{m}$. Dashed lines indicate the junction between epidermis and dermis (d). $h$, hair follicle. (B) Flow cytometry analysis of macrophages isolated from mouse wounds gated for side scatter and F4/80. Expression of M1 and M2 activation markers is shown in RFU (see Methods). Resident skin macrophages were pooled from NS $(n=5)$. ${ }^{*} P<0.05$, ${ }^{\star *} P<0.01$, ${ }^{* \star \star} P<0.001$, Student's $t$ test. (C) Representative macroscopic aspects of wounds at $0,5,7$, and 10 days after wounding. (D) Statistical analysis of 20 wound areas per group, expressed as percentage of the initial wound size (day 0), for iron-dextran-treated mice (filled symbols) in the presence and absence of DFX or etanercept. Open symbols denote PBS-dextran treatment. Results are mean \pm SD $(n=5)$ representing 1 of 3 independent experiments. ${ }^{*} P<0.05$, Mann-Whitney test. (E) Representative photomicrographs of mouse wounds at day 5 after wounding stained for $\mathrm{F} 4 / 80$ and TNF- $\alpha$, with persistent TNF- $\alpha$-producing macrophages (yellow), in iron-loaded, but not PBS-dextran- or etanercept-treated, wounds. Nuclei were stained with DAPI. Scale bars: $100 \mu \mathrm{m}$. Dashed lines indicate the junction between eschar and wound margin. (F) Statistical analysis of 20 wound areas per group for iron-loaded and PBS-dextran control mice treated with clodronate or PBS liposomes. ${ }^{*} P<0.05,{ }^{* \star} P<0.01$, Mann-Whitney test.

contrast to NS taken in sufficient distance from CVUs of the same patients' legs, which showed almost no $\gamma \mathrm{H} 2 \mathrm{AX}$ expression in nuclei of resident fibroblasts (Figure 5A), a high number of fibroblasts adjacent to CVUs stained positive for $\gamma \mathrm{H} 2 \mathrm{AX}$ (Figure 5B). Thus, fibroblasts and not inflammatory cells revealed DNA damage. Western blot analysis of wound lysates confirmed these data (Supplemental Figure 9). Iron-loaded mice, in contrast to PBS-dextran-injected mice, showed enhanced induction of $\gamma \mathrm{H} 2 \mathrm{AX}$ that could be completely prevented by either DFX (Figure 5C) by clodronate liposome depletion of macrophages (data not shown). Immunostaining for p16 ${ }^{\text {INK4a }}$, a robust in vivo marker for aging in murine and human skin $(48,49)$, revealed higher $16^{\mathrm{INK} 4 \mathrm{a}}$ expression in CVUs, but not in NS of the patients' same leg (Figure 5, D and E).

Collectively, our data indicate that the iron-induced activation of the proinflammatory M1 macrophage population contributes to enhanced DNA damage and senescence in skin resident fibroblasts and, hence, distinctly impairs their capacity for tissue repair.

\section{Discussion}

This report defines what we believe to be a previously undescribed iron-induced macrophage population in CVUs in humans and the corresponding murine model, with a unique unrestrained proinflammatory M1 activation profile along with high expression of the M2 iron scavenger receptor CD163, which - via release of TNF- $\alpha$, ONOO ${ }^{*}$, and $\mathrm{OH}^{*}-$ is responsible for the perpetuation of inflammation, tissue breakdown, and impaired wound healing (Figure 6). To identify and characterize this macrophage population in the pathogenesis of macrophage-driven chronic inflammation, we analyzed tissue samples from patients with CVUs, which, in contrast to human AWs, revealed a persistent increase in macrophage numbers with an unrestrained proinflammatory M1 activation state. This macrophage population expressed both classical proinflammatory M1 markers (TNF- $\alpha$, iNOS, IL-12, and CCR2) and M2 markers, particularly the scavenger receptors CD163 and
CD206, and exhibited low expression of IL-4R $\alpha$, IL-10, Dectin-1, CD36, arginase, M2 receptors, and cytokines, which normally terminate the inflammatory response and contribute to angiogenesis, matrix deposition, and remodeling (50).

We show here, for the first time to our knowledge, that the persistence of an unrestrained proinflammatory M1 macrophage population with an incomplete switch to antiinflammatory M2 macrophages eventually led to tissue breakdown and impaired healing in CVUs. We furthermore identified iron to be responsible for the induction of the unrestrained proinflammatory M1 macrophage population. Iron accumulated in macrophages in CVUs and in the corresponding murine model. Normally, iron bound to ferritin is safely stored in macrophages, and, hence, macrophages are essential to prevent iron toxicity $(51,52)$. However, under iron-overloading conditions with persisting erythrocyte extravasation and coinciding leukocyte trapping in the tissue, as in CVUs, persistence of the unrestrained proinflammatory M1 macrophage population may occur. This was in fact the case: we fully reproduced the induction of the unrestrained proinflammatory M1 macrophage population with enhanced CD163 expression, leading to impaired wound healing, in the macrophage iron-overload mouse model. Our findings that the iron chelator DFX prevented induction of the unrestrained proinflammatory M1 macrophage population and the impaired wound-healing phenotype strongly support this notion.

Inflammatory conditions in the ulcer tissue further promote iron retention in macrophages. In fact, exposure of macrophages to bacteria (53), the bacterial wall constituent LPS, and Toll-like receptor agonists (54), all occurring in CVUs $(55,56)$, result in the induction of hepcidin (57), the major inhibitor of iron export from macrophages. In addition, proinflammatory cytokines released at high concentrations from the unrestrained proinflammatory M1 macrophages further enhance the uptake and intracellular iron retention in macrophages by the induction of transmembrane transporter-1 expression (58). In contrast, M2 cytokines like IL-10 - which are virtually absent in the proinflammatory M1 macrophages in nonhealing CVUs - are responsible for the release of iron from the macrophage to the extracellular space (58). Collectively, these CVU-related conditions, in addition to persistent erythrocyte extravasation, further increase the intracellular iron pool, promoting activation of the proinflammatory M1 macrophage population.

Our studies do not allow us to discern whether this unrestrained proinflammatory M1 macrophage population represents a continuum of functional state in the conceptual M1/M2 framework (32). It is also not clear whether phenotypic alteration of this macrophage population is the result of differentiation of the initial macrophages within the tissue or of polarization of a distinct population of macrophages into the wound/ulcer site, where they replace the original macrophages. Regardless of the mechanism, it is likely that an inflammatory environment leads to the exposure of macrophages to several stimuli. We here identified iron overloading in macrophages as the major environmental cue responsible for the persistence of the unrestrained proinflammatory M1 activation state of the wound macrophage population.

The present approach highlighted the usefulness of a more complete assessment of the innate immune response and helped to identify TNF- $\alpha$ as a key effector molecule released from macrophages with unrestrained proinflammatory M1 activation phenotype. This is supported by our finding that the TNF- $\alpha$ antagonist etanercept dampened proinflammatory macrophage 
A
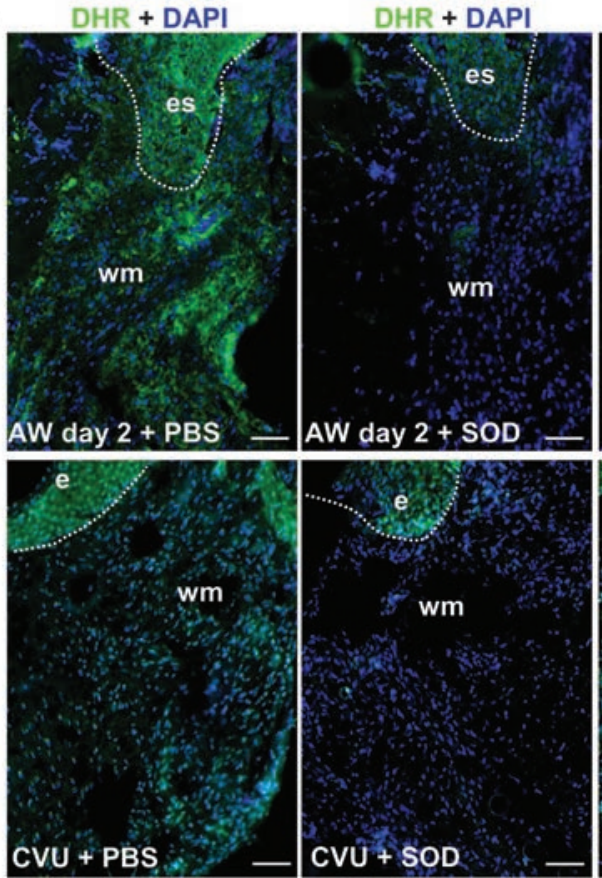

DHR + DAPI
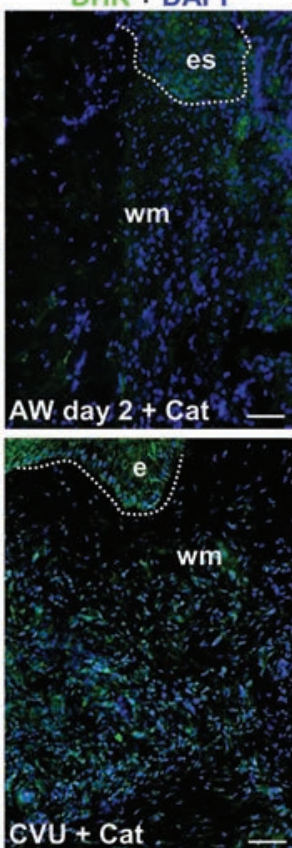

$\mathrm{DHR}+\mathrm{DAPI}$
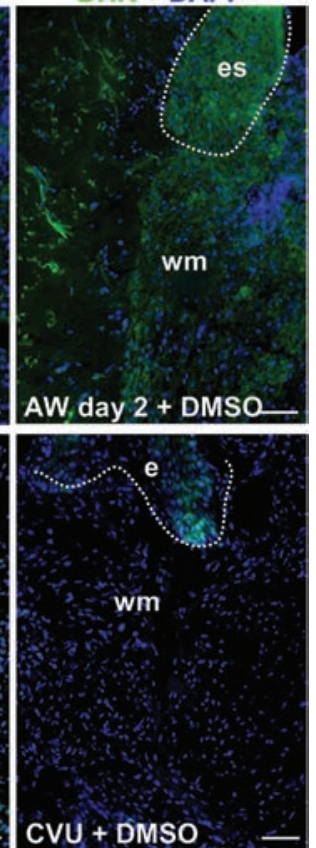

B
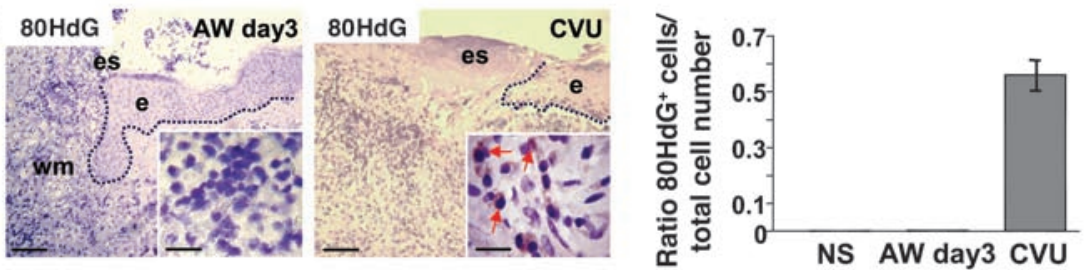

C 3-NT
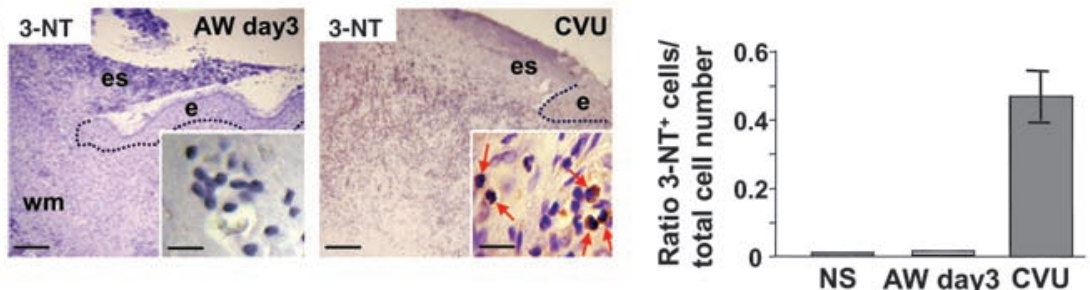

\section{Figure 4}

The iron-induced macrophage population with unrestrained proinflammatory M1 phenotype releases toxic amounts of $\mathrm{OH}^{\cdot}$ and $\mathrm{ONOO}^{\cdot}$ in situ. (A) Representative photomicrographs of skin cryosections derived from wound margins of AWs 2 days after wounding and CVUs. Oxidative burst was detected in cryosections incubated with DHR, a ROS-sensitive dye; thus, ROS concentrations correlated with green fluorescence. Shown are coincubations of cryosections with DHR and SOD, which scavenges $\mathrm{O}_{2}^{--}$, with the $\mathrm{H}_{2} \mathrm{O}_{2}$ scavenger Cat, and with the $\mathrm{OH}^{*}$ scavenger DMSO. Nuclei were stained with DAPI. Scale bars: $150 \mu \mathrm{m}$. (B and C) Representative photomicrographs of paraffin-embedded skin sections from AWs 3 days after wounding and CVUs stained with (B) an antibody against $8 \mathrm{OHdG}$ and (C) an antibody against 3-NT. Dashed lines indicate the junction between epidermis and dermis. Scale bars: $150 \mu \mathrm{m} ; 50 \mu \mathrm{m}$ (insets). Quantification of positive cells per 10 high-power fields, assessed for 10 different sections of 5 different AW and CVU samples, is shown as mean \pm SD ratios of positive to total cells. (B) The brown-colored precipitate is indicative of oxidative DNA damage in CVUs (inset, arrows), but not AWs. (C) Increased protein nitration was observed in CVUs, but not AWs. (D) Nitroblot analysis of wound lysates with an antibody against 3-NT from PBS-treated mice and from iron-loaded mice and iron-loaded mice treated with DFX, etanercept, or clodronate equilibrated to actin. Positive bands indicate increased levels of protein nitration in iron-loaded wounds compared with reduced levels in irondextran-loaded mice treated with DFX, etanercept, or clodronate.

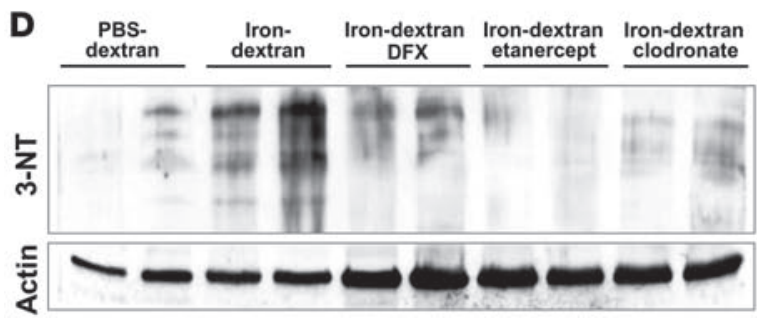

activation and rescued delayed healing in the murine iron-overload model, whereas injection of rTNF- $\alpha$ in the margins of AWs significantly impaired healing in control mice.

As to the mechanism by which iron stimulates continuous macrophage activation, there is evidence from 2 independent reports that intracellular iron activates $\mathrm{NF}-\kappa \mathrm{B}$, the major transcription factor of genes including, among others, proinflammatory TNF- $\alpha$ in a model of LPS-stimulated (59) or iron-loaded rat liver macrophages (60).
Apart from maintaining macrophage activation (41, 42), TNF- $\alpha$ tilts the metabolism of connective tissue fibroblasts toward proteolysis (61) and enhances expression of iNOS, which was highly upregulated in unrestrained proinflammatory M1 activated macrophages in CVUs. iNOS in turn stimulates macrophage generation of $\mathrm{NO}^{*}$, which, together with $\mathrm{O}_{2}^{-}$, forms ONOO' and leads to nitrative tissue damage. We showed that redox-active iron - via the Fenton reaction - generated the highly toxic $\mathrm{OH}^{\bullet}$ in sections from CVU biopsies; in addition, it stimulated macrophages to produce 


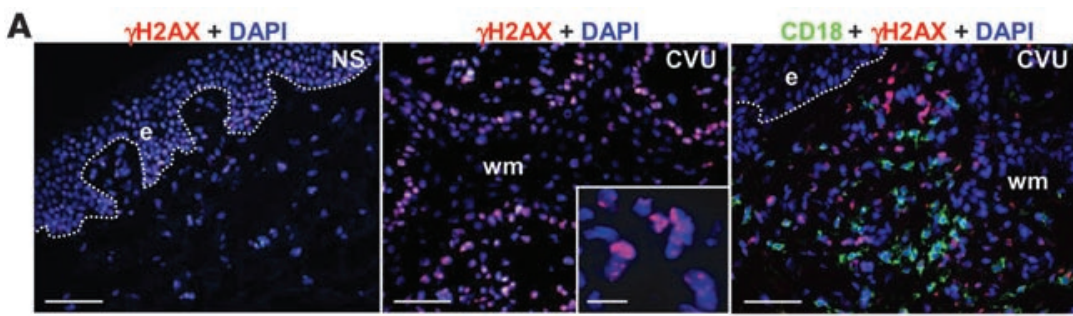

B

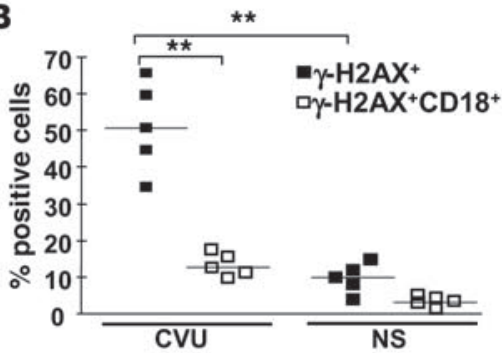

D

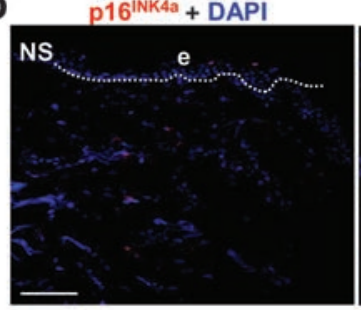

$\mathrm{p} 16^{\mathrm{INK} 4 \mathrm{a}}+\mathrm{DAPI}$

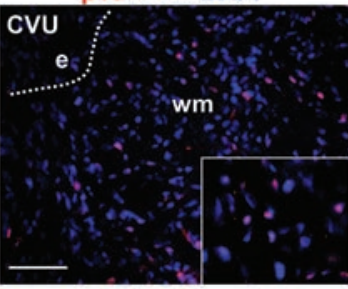

C

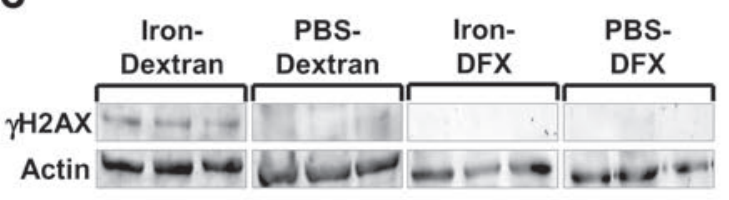

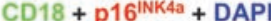

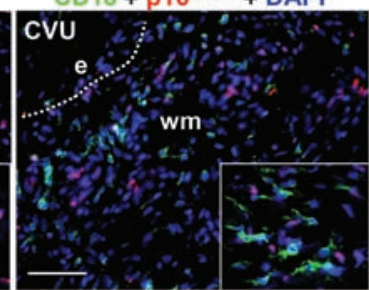

E

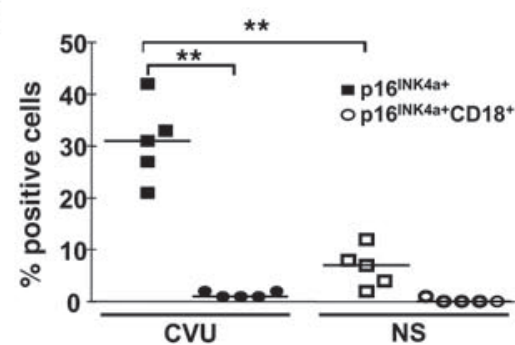

Figure 5

Enhanced ROS release by the unrestrained proinflammatory M1 activated macrophage population activates a senescence program in resident wound fibroblasts. (A) Representative photomicrographs of cryosections from wound margins from CVUs and NS with increased numbers of $\gamma \mathrm{H} 2 \mathrm{AX}+$ foci in the cell nuclei of wound margins. Double staining for $\gamma \mathrm{H} 2 \mathrm{AX}$ and the leukocyte marker CD18 revealed exclusive $\gamma \mathrm{H} 2 \mathrm{AX}$ expression in wound-adjacent fibroblasts. Nuclei were stained with DAPI. Scale bars: $150 \mu \mathrm{m} ; 50 \mu \mathrm{m}$ (inset). (B) Quantification of $\gamma \mathrm{H} 2 \mathrm{AX}+$ and $\gamma \mathrm{H} 2 \mathrm{AX}+\mathrm{CD} 18^{+}$cells in 10 high-power fields from CVUs and NS samples $(n=5)$. Results are mean \pm SD percent positive cells relative to total cells. ${ }^{* \star} P<0.01$, Student's $t$ test. (C) Representative Western blot of wound lysates from iron-loaded and PBS control wounds with and without treatment with DFX, equilibrated to total actin, showing expression of $\gamma \mathrm{H} 2 \mathrm{AX}$ in iron-loaded, but not control or DFX-treated, wounds $(n=3)$. (D) Representative photomicrographs of cryosections of wound margins and NS derived from CVU patients showing increased numbers of p16INK4a in the cell nuclei of ulcer margins. Double staining for $\mathrm{p} 16^{\mathrm{INK} 4 \mathrm{a}}$ and CD18 showed $\mathrm{p} 16^{\mathrm{INK} 4 \mathrm{a}}$ expression exclusively in wound-adjacent fibroblasts, not in CD18+ cells. Nuclei were stained with DAPI. Scale bars: $150 \mu \mathrm{m}$. Original magnification of insets, $\times 400$. (E) Quantification of p16 INK4a+ and p16INK4a+CD18+ cells in 10 high-power fields from 5 different CVUs and NS samples. Results are mean \pm SD percent positive cells relative to total cells. ${ }^{* *} P<0.01$, Student's $t$ test.

enhanced $\mathrm{ONOO}^{*}$ concentrations. Both $\mathrm{OH}^{*}$ and $\mathrm{ONOO}^{*}$ can lead to DNA double strand breaks and to the installment of the $16^{\text {INK4a }}$ dependent senescence program in ulcer-adjacent fibroblasts. p16 $6^{\text {INK4a }}$ functions as an inhibitor of the D-type cyclin-dependent kinase CDK4 and is known to arrest cells in the $\mathrm{G}_{1}$ phase of the cell cycle, implementing irreversible growth arrest. Our in vivo data corroborated and extended previous in vitro data that ulcer-derived fibroblast cultures undergo accelerated replicative senescence (62). Although an increase in telomere erosion as a consequence of the pro-oxidant environment of ulcer resident fibroblasts was previously excluded (62), we showed here that accelerated aging of ulcer resident fibroblasts was rather caused by the installment of a p16 ${ }^{\text {INK } 4 a}$-dependent senescence program in vivo. Interestingly, replicative senescent ulcer resident fibroblasts in CVUs revealed an impaired ability to produce their correct stromal address code (a specific combination of released chemokines and cytokines; ref. 63), which is essentially required to generate a physiological self-terminating inflammatory response (62). Independent of this interesting observation, we here identified iron-induced unrestrained proinflammatory M1 macrophages to be upstream of the reported dysfunctional and senescent ulcer fibroblasts. In fact, treatment with clodronate liposomes and DFX prevented induction of $\gamma \mathrm{H} 2 \mathrm{AX}$, a recognized in vivo aging marker (47), in fibroblasts of AWs from iron-dextran-treated mice.

Apart from initiating a senescence program in resident fibroblasts, high ROS concentrations released by proinflammatory macrophages or other antigen-presenting cells may stimulate $T$ cells that suppress wound healing in CVUs. In fact, recent reports have shown that appropriate ROS signaling in antigenpresenting cells is required to suppress Th1-type autoimmune responses (64) and to induce Th2 immunity to allergens (65). It remains to be elucidated whether similar suppressive or Th2 lymphocytes, or rather IFN- $\gamma$-producing Th1 T cells, occur in the highly pro-oxidative ulcus environment.

Our findings have substantial clinical impact, as potential therapeutic targets have been identified in the specific pathogenic context of CVUs. First, iron is a well-known suspect in oxidative 


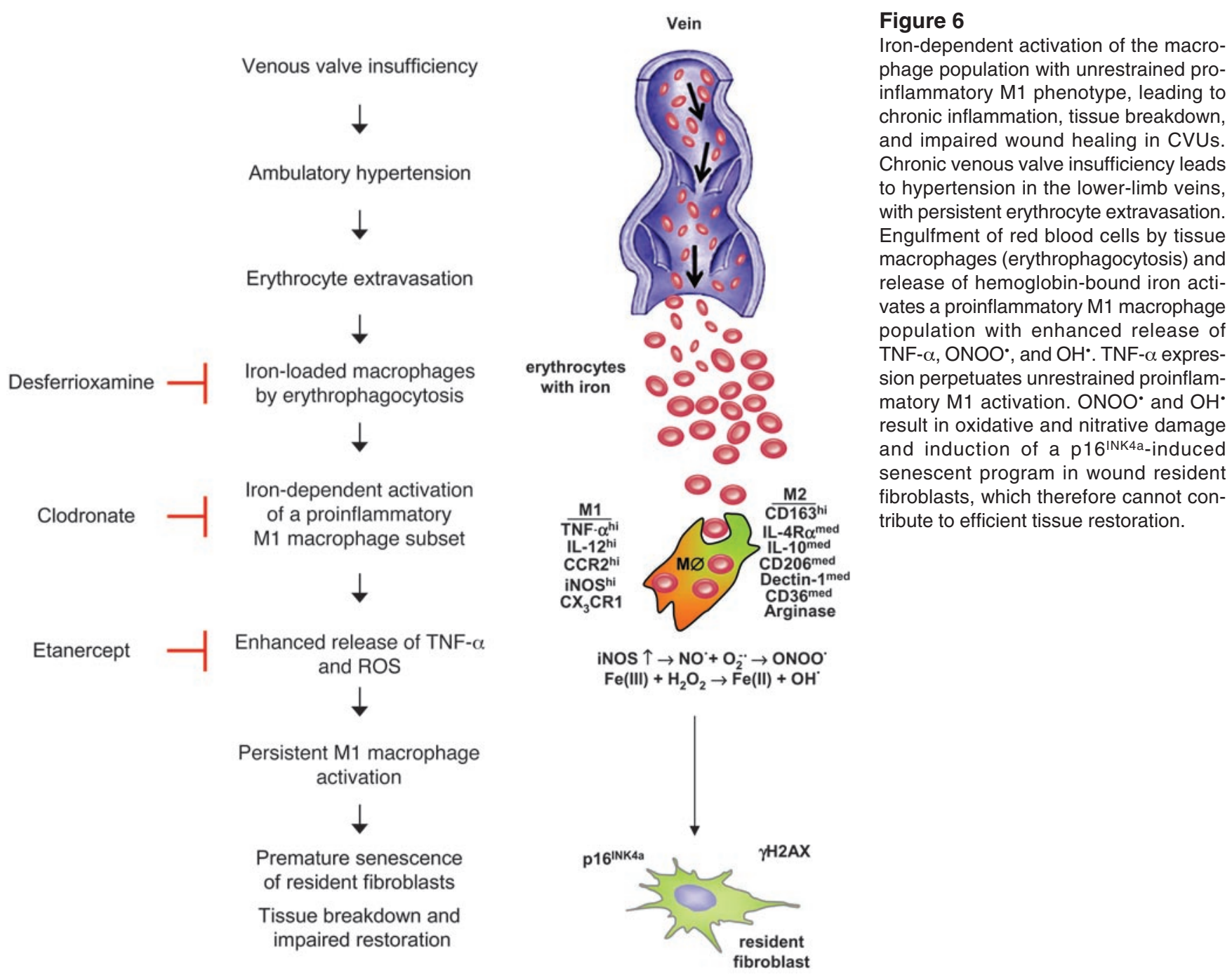

tissue damage (9); however, to our knowledge, it has never been recognized as causal for persistent macrophage-driven inflammation in CVUs. Thus, we believe our data on the critical role of macrophage iron overload in preventing the physiologic switch from M1 to M2 macrophages to be novel. We found that DFX, the most specific and potent iron chelator, fully prevented generation of the proinflammatory M1 macrophage population and consequently prevented impaired wound healing in the murine iron-overload model. Interestingly, experimental and clinical studies demonstrate that iron chelation by DFX substantially protects myocardial fibers against iron-dependent damage in iron-overload conditions like $\beta$-thalassemia (66) and decreases necrosis in dorsally localized skin flaps (67). Previously, DFX covalently bound to cellulose to selectively pick up iron was reported to abrogate lipid peroxidation in human dermal fibroblasts in vitro (19), but this has not been assessed in CVUs in vivo, and the effect of DFX on macrophages when topically applied is currently not known. Second, our in situ data showed that $\mathrm{O}_{2}^{-\bullet}$ and $\mathrm{OH}^{\bullet}$ increased in CVUs. Notably, topical application of DMSO, an $\mathrm{OH}^{*}$ scavenger, or of allopurinol, which exerts its effect by inhibition of xanthine oxidase, which itself generates high $\mathrm{O}_{2}{ }^{\bullet}$ concentrations under the hypoxic conditions of CVUs, have been reported to improve CVU healing in a series of
133 studied patients (68). Third, we found that iron induced a macrophage population with unrestrained proinflammatory M1 activation pattern and only moderate upregulation of M2 scavenger receptors (CD163 and CD206). It will be of major importance to search for small molecular therapeutics that induce a switch to antiinflammatory macrophage populations (69). Among many agonists, glucocorticoids have been shown to promote antiinflammatory macrophage populations (70). Interestingly, treatment of CVUs with topical corticosteroids was of benefit for $79 \%$ of the patients in terms of healing (71). It remains to be seen whether the favorable outcome following short-term topical application of corticosteroids in fact results in a switch from proinflammatory macrophages to macrophage populations with antiinflammatory properties. Fourth, we found that the release of high concentrations of TNF- $\alpha$ from the proinflammatory M1 macrophages perpetuated inflammation. Interestingly, topical application of infliximab, a chimeric IgG1 monoclonal antibody that binds with high affinity and specificity to the soluble and transmembrane TNF- $\alpha$, resulted in significant improvement of healing in 12 of 14 previously therapy-resistant CVUs (12). Because of the impressive success of anti-TNF- $\alpha$ therapies in a variety of conditions (72-74), including the aforementioned study on CVUs (12), the next gener- 
ation of small molecules targeting different steps of TNF- $\alpha$ signaling have been developed, some of which are currently being tested in clinical trials (75). Finally, a complementary analysis of human subjects and a relevant mouse model was essential for establishing a role for the iron-dependent induction of a proinflammatory M1 macrophage population and effector molecules, such as TNF- $\alpha$, $\mathrm{ONOO}^{\circ}$, and $\mathrm{OH}^{*}$, that drive chronic inflammation, tissue breakdown, premature aging, and ulcer formation.

Thus far, the concept of the M1/M2 dichotomy was defined in vitro; however, apart from few studies on acute tissue damage models in mice $(30,76-78)$, it has not to our knowledge been studied in macrophage-driven chronic inflammatory disease in humans. The identification of iron accumulation in macrophages as the prime event in the complex pathogenesis of CVUs eventually driving persistent inflammation and tissue damage is also of major relevance for other chronic inflammatory disorders with enhanced iron storage in macrophages, like arteriosclerosis, multiple sclerosis, and other neurodegenerative diseases (79-82). Taken together, our findings provide powerful insight into the role of an iron-induced macrophage population in vivo. Targeting this population holds substantial promise for the development of novel therapies for these difficult-to-treat conditions.

\section{Methods}

Collection of human tissue samples. We studied 16 patients with nonhealing ulcers (CEAP classification C6; ref. 83) that, despite conventional therapy, persisted for more than 4 months. After informed consent was obtained, 5-mm biopsies were taken from the ulcer edge and from healthy skin of the same patient's leg. AWs of $5 \mathrm{~mm}$ were inflicted on the upper thighs of healthy volunteers. At 1, 2, and 5 days after wounding, a second 5 -mm biopsy was punched around the initially inflicted wounds. All protocols were approved by the Ethics Committee of the University of Ulm (protocol nos. 224/2002, 225/2002, and 170/2009) according to the Declaration of Helsinki Principles.

Immunohistochemistry. Sections $(5 \mu \mathrm{m})$ from paraffin-embedded tissue were subjected to antigen retrieval with Proteinase K (Dako) and incubated with anti-CD68 (PG-M1; Dako), anti-3-NT (1A6; Millipore) and anti-8OHdG (15A3; eBioscience) antibodies and HRP-conjugated secondary antibodies (Dako). Peroxidase activity was visualized using diaminobenzidine (Sigma-Aldrich). For iron staining, cryosections were incubated with Perl Prussian solution (1:1, 5\% potassium ferrocyanide and 5\% $\mathrm{HCl}$ ) (Merck) and with Nuclear Fast Red (ROTH).

Immunofluorescence staining. Cryosections $(5 \mu \mathrm{m})$ were incubated with antibodies against human iNOS (BD Transduction Laboratories), TNF- $\alpha$ (abcam), CD163/M130 (GHI/61; Santa Cruz Biotechnology), arginase-1 (M-20; Santa Cruz Biotechnology), IL-12p40 (1-1A4; GeneTex), CD18 (YFC118.3; GeneTex), CD206/MMR (19.2; BD Biosciences - Pharmingen), $\gamma \mathrm{H} 2 \mathrm{AX}$ (9F3; abcam) and p16 ${ }^{\mathrm{INK} 4 \mathrm{a}}$ (16P04; Neomarkers) and mouse F4/80 (BM8; eBioscience), GR-1 (RB6-8C5; eBioscience), CD4 (L3T4; eBioscience), CD8 (53-6.7; eBioscience), CD117 (ACK2; eBioscience), CD205 (NLDC145 ; AbD Serotec), TNF- $\alpha$ (MP6-XT22; BD Biosciences - Pharmingen and abcam), CD206 (MR5D3; Santa Cruz Biotechnology), arginase-1 (M-20; Santa Cruz Biotechnology), CD207 (Langerin; Santa Cruz Biotechnology), and IL-12 (C15.6; GeneTex). Isotype Ig served as negative control, and Alexa Fluor 488 and Alexa Fluor 555 (Molecular Probes) served as secondary antibodies. Photomicrographs were produced using a Zeiss Axiophot microscope and corresponding software (Zeiss).

Qualitative assessment of ROS in situ. Fresh wound cryosections (10 $\mu \mathrm{m})$ were incubated with $10 \mu \mathrm{M}$ DHR for 20 minutes at $37^{\circ} \mathrm{C}$, resulting in a ROS-dependent increase in green fluorescence. For ROS scavenging experiments, serial cryosections were coincubated with DHR and either SOD (100 U/ml; Sigma-Aldrich), catalase (1,000 U/ml; MBL Int.), or 0.2\% DMSO (Merck). Photomicrographs were taken immediately, with identical exposure time for all settings.

FACS analysis. Cell suspensions isolated from fresh tissue by enzymatic digestion (28) or in vitro matured macrophages were incubated with antihuman CD206-Alexa Fluor 647 (15-2; BioLegend), CCR2-Alexa Fluor 647 (TG5/CCR2; BioLegend), CD163-PerCP/Cy5.5 (RM3/1; BioLegend), IL-4R $\alpha$-fluorescein (25463; R\&D Systems), Dectin-1/CLEC7A-PE (259931; R\&D Systems), CX3CR1 (Lifespan Biosciences), and CD36-FITC (NL07; eBioscience) antibodies or anti-mouse CCR2 (abcam), F4/80-Alexa Fluor 488 (eBioscience), F4/80-Pacific blue (BM8; eBioscience), Ly-6C-PerCP/ Cy5.5C (HK1.4; eBioscience), CD163 (M-96; Santa Cruz Biotechnology), IL-4R $\alpha / C D 124-P E$ (mIL4R-MI; BD Biosciences - Pharmingen), Dectin-1 (2A11; AbD Serotec), CD204-PE/SRA I (2F8; AbD Serotec), CD301/MGL1,2 (ER-MP23; AbD Serotec), CD206-Alexa Fluor 647 (MR5D3; BioLegend), or isotype Ig. Intracellular markers were identified after permeabilization (eBioscience) with anti-human IL-12/IL-23p40-Alexa Fluor 488 (C11.5; BioLegend), CD68-PE and CD68-FITC (Y1/82A; eBioscience), TNF- $\alpha$-PerCP-Cy5.5 (MAb11; eBioscience), and IL-10-Pacific blue (JES3-9D7; eBioscience) and with anti-mouse TNF- $\alpha$-PerCP/Cy5.5 (MP6-XT22; BioLegend) and IL-12/ IL-23p40-PerCP-Cy5.5 (C17.8; eBioscience) or isotype Ig. Cells were measured with a BD FACS Canto II flow cytometer (BD Biosciences) and analyzed with FlowJo Software (Tree Star Inc.). Marker expression is given as relative fluorescent units (RFU) representing the ratio of the geometric MFI from the specific antibody to that of the isotype-matched antibody.

Western blotting. Frozen crushed tissue was dissolved in ice-cold lysis buffer (50 mM Tris-Cl, pH 7.6; $150 \mathrm{mM} \mathrm{NaCl}$; 1\% NP-40; and protease inhibitors [Roche]) and cleared by centrifugation. Equal amounts of protein were resolved by SDS-PAGE (Bio-Rad) and transferred to nitrocellulose membranes (Schleicher \& Schuell). Membranes were blocked with 5\% BSA/TBS/ Tween and incubated with antibodies against $\gamma \mathrm{H} 2 \mathrm{AX}$ (9F3; Assay DesignsStressgen), 3-NT (1A6; Millipore) and actin (Calbiochem). Oxyblots were performed using the Oxyblot Protein Oxidation Detection Kit (Millipore) according to the manufacturer's instructions.

Human PBMC-derived primary macrophages. Primary macrophages were obtained as described previously (27). Monocytes were purified from buffy coats by density gradient using Lymphocyte Preparation Medium 1077 (PAA) followed by CD14+ magnetic cell sorting (Miltenyi Biotech). Cells were cultured in DMEM with $2 \mathrm{mM}$ L-glutamine and $50 \mathrm{mg} / \mathrm{ml}$ gentamicin (Biochrom) at $37^{\circ} \mathrm{C}, 5 \% \mathrm{CO}_{2}$, for 9 days. Where indicated, macrophages were coincubated for the last 4 days with the combination $500 \mathrm{U} / \mathrm{ml}$ IFN- $\gamma$ (PeproTech) and $10 \mathrm{ng} / \mathrm{ml}$ LPS (Sigma-Aldrich) or the combination $0.1 \mu \mathrm{M}$ dexamethasone (Merck) and $10 \mathrm{ng} / \mathrm{ml} \mathrm{IL-4} \mathrm{(PeproTech).} \mathrm{For} \mathrm{Fenton} \mathrm{chem-}$ istry, macrophages were subjected to $10 \mu \mathrm{M} \mathrm{Fe}(\mathrm{III})$-chloride, $10 \mu \mathrm{M}$ ascorbate, and $50 \mu \mathrm{M} \mathrm{H}_{2} \mathrm{O}_{2}$ (Sigma-Aldrich).

Mice and wound-healing model. All experiments were performed with 8- to 12-week-old C57BL/6 mice in compliance with the German Law for Welfare of Laboratory Animals and were approved by the Institutional Review Board of the University of Ulm.

Mice were treated intraperitoneally with $5 \mathrm{mg}$ iron-dextran at $200 \mu \mathrm{l} /$ mouse or $200 \mu$ l PBS-dextran (Sigma-Aldrich) every 3 days for 21 days. Selected groups were treated, following the same schedule, with $6 \mathrm{mg}$ per $200 \mu \mathrm{l}$ DFX (Sigma-Aldrich) intraperitoneally. Etanercept (Wyeth) was injected at $25 \mu \mathrm{g} /$ wound into wound margins at days 3, 4, 5, and 6 after wounding; rTNF- $\alpha$ (PeproTech) was administered at 5,000 U/ wound at days 2,3 , and 4 ; clodronate or PBS liposomes were injected at $12.5 \mathrm{mg} /$ wound at day 4 after wounding. 46 - $\mathrm{mm}$ full-thickness excisional wounds were produced under anesthesia on both sides of the shaved back of each mouse, as described previously $(27,28)$. At the indi- 
cated time points, wounds were photographed, and wound areas were quantified using Adobe Photoshop 7.0.1 software (Adobe Systems).

Statistics. Quantitative data are presented as mean \pm SD. Statistical significance was determined by 2 -tailed Student's $t$ test, or Mann-Whitney $U$ test in cases of non-Gaussian distribution. $P$ values less than 0.05 were considered statistically significant.

\section{Acknowledgments}

We thank Natalie Gall for technical assistance and Siegfried Reihle for creating the schematic drawing in Figure 6. We are grateful to Hartmut Geiger for critically reading the manuscript. This study was supported by the DFG (SFB 497 and KFO 142 to K. Scharffetter-Kochanek; SU 195/3-1 to C. Sunderkötter) and by the Integrated Project CASCADE (HEALTH - F5-2009-223236 to
K. Scharffetter-Kochanek) of the European Union. A. Sindrilaru was supported by grant Bausteinförderung 3.2 from the Medical Faculty, University of Ulm. We are grateful for the financial support of the Baden-Württemberg Stiftung in the program Adulte Stamzellen II (P-BWS-ASII/15).

Received for publication July 24, 2010, and accepted in revised form December 8, 2010.

Address correspondence to: Karin Scharffetter-Kochanek, Department of Dermatology and Allergic Diseases, University of Ulm, Maienweg 12, 89081 Ulm, Germany. Phone: 49.731.500.57501; Fax: 49.731.500.57502; E-mail: karin.scharffetter-kochanek@ uniklinik-ulm.de.
1. Bergan JJ, Schmid-Schönbein GW, Smith PD, Nicolaides AN, Boisseau MR, Eklof B. Chronic venous disease. NEngl J Med. 2006;355(5):488-498.

2. Raju S, Neglen P. Clinical practice. Chronic venous insufficiency and varicose veins. N Engl J Med. 2009; 360(22):2319-2327.

3. Margolis DJ, Bilker W, Santanna J, Baumgarten M. Venous leg ulcer: incidence and prevalence in the elderly. J Am Acad Dermatol. 2002;46(3):381-386.

4. Singer AJ, Clark RA. Cutaneous wound healing. NEngl J Med. 1999;341(10):738-746.

5. Agren MS, et al. Causes and effects of the chronic inflammation in venous leg ulcers. Acta Derm Venereol Suppl (Stockh). 2000;210:3-17.

6. Smith PD. Update on chronic-venous-insufficiency-induced inflammatory processes. Angiology. 2001;52 suppl 1:S35-S42.

7. Dow G, Browne A, Sibbald RG. Infection in chronic wounds: controversies in diagnosis and treatment. Ostomy Wound Manage. 1999;45(8):23-27.

8. Robson MC. Wound infection. A failure of wound healing caused by an imbalance of bacteria. Surg Clin North Am. 1997;77(3):637-650.

9. Wlaschek M, Scharffetter-Kochanek K. Oxidative stress in chronic venous leg ulcers. Wound Repair Regen. 2005;13(5):452-461.

10. Rosner K, Ross C, Karlsmark T, Petersen AA, Gottrup F, Vejlsgaard GL. Immunohistochemical characterization of the cutaneous cellular infiltrate in different areas of chronic leg ulcers. Apmis. 1995; 103(4):293-299.

11. Abd-El-Aleem SA, et al. Expression of nitric oxide synthase isoforms and arginase in normal human skin and chronic venous leg ulcers. J Pathol. 2000; 191(4):434-442.

12. Streit M, Beleznay Z, Braathen LR. Topical application of the tumour necrosis factor-alpha antibody infliximab improves healing of chronic wounds. Int Wound J. 2006;3(3):171-179

13. Coleridge Smith PD, Thomas P, Scurr JH, Dormandy JA. Causes of venous ulceration: a new hypothesis. Br Med J (Clin Res Ed). 1988;296(6638)1726-1727.

14. Takase S, Schmid-Schonbein G, Bergan JJ. Leukocyte activation in patients with venous insufficiency. J Vasc Surg. 1999;30(1):148-156.

15. Weyl A, Vanscheidt W, Weiss JM, Peschen M, Schopf E, Simon J. Expression of the adhesion molecules ICAM-1, VCAM-1, and E-selectin and their ligands VLA-4 and LFA- 1 in chronic venous leg ulcers. $J \mathrm{Am}$ Acad Dermatol. 1996;34(3):418-423.

16. Tarnuzzer RW, Schultz GS. Biochemical analysis of acute and chronic wound environments. Wound Repair Regen. 1996;4(3):321-325

17. Wysocki AB, Staiano-Coico L, Grinnell F. Wound fluid from chronic leg ulcers contains elevated levels of metalloproteinases MMP-2 and MMP-9. J Invest Dermatol. 1993;101(1):64-68.

18. Yeoh-Ellerton S, Stacey MC. Iron and 8-isoprostane levels in acute and chronic wounds. J Invest Derma- tol. 2003;121(4):918-925.

19. Wenk J, et al. Selective pick-up of increased iron by deferoxamine-coupled cellulose abrogates the irondriven induction of matrix-degrading metalloproteinase 1 and lipid peroxidation in human dermal fibroblasts in vitro: a new dressing concept. J Invest Dermatol. 2001;116(6):833-839.

20. Lauer G, et al. Expression and proteolysis of vascular endothelial growth factor is increased in chronic wounds. J Invest Dermatol. 2000;115(1):12-18.

21. Wlaschek M, Peus D, Achterberg V, Meyer-Ingold W, Scharffetter-Kochanek K. Protease inhibitors protect growth factor activity in chronic wounds. BrJ Dermatol. 1997;137(4):646.

22. Chen SM, Ward SI, Olutoye OO, Diegelmann RF, Kelman Cohen I. Ability of chronic wound fluids to degrade peptide growth factors is associated with increased levels of elastase activity and diminished levels of proteinase inhibitors. Wound Repair Regen. 1997;5(1):23-32.

23. Zamboni $\mathrm{P}$, et al. The overlapping of local iron overload and HFE mutation in venous leg ulcer pathogenesis. Free Radic Biol Med. 2006;40(10):1869-1873.

24. Zamboni P, et al. Hemochromatosis C282Y gene mutation increases the risk of venous leg ulceration. J Vasc Surg. 2005;42(2):309-314.

25. Ackerman Z, Seidenbaum M, Loewenthal E, Rubinow A. Overload of iron in the skin of patients with varicose ulcers. Possible contributing role of iron accumulation in progression of the disease. Arch Dermatol. 1988;124(9):1376-1378.

26. Leibovich SJ, Ross R. The role of the macrophage in wound repair. A study with hydrocortisone and antimacrophage serum. Am J Pathol. 1975;78(1):71-100.

27. Peters T, et al. Wound-healing defect of CD $18(-/-)$ mice due to a decrease in TGF-beta1 and myofibroblast differentiation. EMBO J. 2005;24(19):3400-3410.

28. Sindrilaru A, et al. Wound healing defect of Vav3 $3^{-/}$ mice due to impaired \{beta\}2-integrin-dependent macrophage phagocytosis of apoptotic neutrophils. Blood. 2009;113(21):5266-5276.

29. Nagaoka T, et al. Delayed wound healing in the absence of intercellular adhesion molecule- 1 or Lselectin expression. Am J Pathol. 2000;157(1):237-247.

30. Lucas $\mathrm{T}$, et al. Differential roles of macrophages in diverse phases of skin repair. J Immunol. 2010; 184(7):3964-3977.

31. Gordon S, Taylor PR. Monocyte and macrophage heterogeneity. Nat Rev Immunol. 2005;5(12):953-964.

32. Mosser DM, Edwards JP. Exploring the full spectrum of macrophage activation. Nat Rev Immunol. 2008;8(12):958-969.

33. Mantovani A, Sica A, Sozzani S, Allavena P, Vecchi A, Locati M. The chemokine system in diverse forms of macrophage activation and polarization. Trends Immunol. 2004;25(12):677-686.

34. Goerdt S, Orfanos CE. Other functions, other genes: alternative activation of antigen-presenting cells. Immunity. 1999;10(2):137-142.
35. Gordon S. Alternative activation of macrophages. Nat Rev Immunol. 2003;3(1):23-35.

36. Dale DC, Boxer L, Liles WC. The phagocytes: neutrophils and monocytes. Blood. 2008;112(4):935-945.

37. Martin P. Wound healing--aiming for perfect skin regeneration. Science. 1997;276(5309):75-81.

38. O'Shea JJ, Murray PJ. Cytokine signaling modules in inflammatory responses. Immunity. 2008; 28(4):477-487.

39. Martinez FO, Gordon S, Locati M, Mantovani A. Transcriptional profiling of the human monocyteto-macrophage differentiation and polarization: new molecules and patterns of gene expression. J Immunol. 2006;177(10):7303-7311.

40. Borda JT, et al. CD163, a marker of perivascular macrophages, is up-regulated by microglia in simian immunodeficiency virus encephalitis after haptoglobin-hemoglobin complex stimulation and is suggestive of breakdown of the blood-brain barrier. Am J Pathol. 2008;172(3):725-737.

41. Wang $\mathrm{H}$, et al. Activated macrophages are essential in a murine model for $\mathrm{T}$ cell-mediated chronic psoriasiform skin inflammation. J Clin Invest. 2006; 116(8):2105-2114.

42. Clarke Moloney M, Lyons GM, Breen P, Burke PE, Grace PA. Haemodynamic study examining the response of venous blood flow to electrical stimulation of the gastrocnemius muscle in patients with chronic venous disease. Eur J Vasc Endovasc Surg. 2006;31(3):300-305.

43. Van Rooijen N, Kors N, vd Ende M, Dijkstra CD. Depletion and repopulation of macrophages in spleen and liver of rat after intravenous treatment with liposome-encapsulated dichloromethylene diphosphonate. Cell Tissue Res. 1990;260(2):215-222.

44. Szabo C, Ischiropoulos H, Radi R. Peroxynitrite: biochemistry, pathophysiology and development of therapeutics. Nat Rev Drug Discov. 2007;6(8):662-680.

45. d'Adda di Fagagna F, et al. A DNA damage checkpoint response in telomere-initiated senescence. Nature. 2003;426(6963):194-198.

46. Garinis GA, et al. Persistent transcription-blocking DNA lesions trigger somatic growth attenuation associated with longevity. Nat Cell Biol. 2009; 11(5):604-615.

47. Herbig U, Ferreira M, Condel L, Carey D, Sedivy JM. Cellular senescence in aging primates. Science. 2006;311(5765):1257.

48. Krishnamurthy J, et al. Ink4a/Arf expression is a biomarker of aging. J Clin Invest. 2004;114(9):1299-1307.

49. Ressler S, et al. p16INK4A is a robust in vivo biomarker of cellular aging in human skin. Aging Cell. 2006;5(5):379-389.

50. Duffield JS. The inflammatory macrophage: a story of Jekyll and Hyde. Clin Sci (Lond). 2003;104(1):27-38.

51. Sullivan JL, Zacharski LR. Hereditary haemochromatosis and the hypothesis that iron depletion protects against ischemic heart disease. Eur J Clin Invest. 2001;31(5):375-377. 
52. Ong WY, Halliwell B. Iron, atherosclerosis, and neurodegeneration: a key role for cholesterol in promoting iron-dependent oxidative damage? Ann NY Acad Sci. 2004;1012:51-64.

53. Peyssonnaux C, Zinkernagel AS, Datta V, Lauth X, Johnson RS, Nizet V. TLR4-dependent hepcidin expression by myeloid cells in response to bacterial pathogens. Blood. 2006;107(9):3727-3732.

54. Theurl I, et al. Autocrine formation of hepcidin induces iron retention in human monocytes. Blood. 2008;111(4):2392-2399.

55. Bjarnsholt $\mathrm{T}$, et al. Why chronic wounds will not heal: a novel hypothesis. Wound Repair Regen. 2008;16(1):2-10.

56. Pukstad BS, et al. Non-healing is associated with persistent stimulation of the innate immune response in chronic venous leg ulcers.J Dermatol Sci. 2010;59(2):115-122.

57. De Domenico I, McVey Ward D, Kaplan J. Regulation of iron acquisition and storage: consequences for iron-linked disorders. Nat Rev Mol Cell Biol. 2008;9(1):72-81.

58. Ludwiczek S, Aigner E, Theurl I, Weiss G. Cytokinemediated regulation of iron transport in human monocytic cells. Blood. 2003;101(10):4148-4154.

59. Xiong S, et al. Signaling role of intracellular iron in NF-kappaB activation. J Biol Chem. 2003; 278(20):17646-17654.

60. Chen L, Xiong S, She H, Lin SW, Wang J, Tsukamoto H. Iron causes interactions of TAK1, p21ras, and phosphatidylinositol 3-kinase in caveolae to activate IkappaB kinase in hepatic macrophages. J Biol Chem. 2007;282(8):5582-5588.

61. Scharffetter K, et al. Synergistic effect of tumor necrosis factor-alpha and interferon-gamma on collagen synthesis of human skin fibroblasts in vitro. Exp Cell Res. 1989;181(2):409-419.

62. Wall IB, et al. Fibroblast dysfunction is a key fac- tor in the non-healing of chronic venous leg ulcers. J Invest Dermatol. 2008;128(10):2526-2540.

63. Parsonage $G$, et al. A stromal address code defined by fibroblasts. Trends Immunol. 2005;26(3):150-156.

64. Gelderman KA, et al. Macrophages suppress $\mathrm{T}$ cell responses and arthritis development in mice by producing reactive oxygen species. J Clin Invest. 2007;117(10):3020-3028.

65. Tang $\mathrm{H}$, et al. The $\mathrm{T}$ helper type 2 response to cysteine proteases requires dendritic cell-basophil cooperation via ROS-mediated signaling. Nat Immunol. 2010;11(7):608-617.

66. Grisaru D, Goldfarb AW, Gotsman MS, Rachmilewitz EA, Hasin Y. Deferoxamine improves left ventricular function in beta-thalassemia. Arch Intern Med. 1986;146(12):2344-2349.

67. Weinstein GS, Maves MD, McCormack ML. Deferoxamine decreases necrosis in dorsally based pig skin flaps. Otolaryngol Head Neck Surg. 1989; 101(5):559-561.

68. Salim AS. The role of oxygen-derived free radicals in the management of venous (varicose) ulceration: a new approach. World J Surg. 1991; 15(2):264-269.

69. Gerber JS, Mosser DM. Reversing lipopolysaccharide toxicity by ligating the macrophage Fc gamma receptors. J Immunol. 2001;166(11):6861-6868.

70. Liu Y, et al. Glucocorticoids promote nonphlogistic phagocytosis of apoptotic leukocytes. JImmunol. 1999;162(6):3639-3646

71. Hofman D, Moore K, Cooper R, Eagle M, Cooper S. Use of topical corticosteroids on chronic leg ulcers. $J$ Wound Care. 2007;16(5):227-230.

72. Lipsky PE, et al. Infliximab and methotrexate in the treatment of rheumatoid arthritis. Anti-Tumor Necrosis Factor Trial in Rheumatoid Arthritis with Concomitant Therapy Study Group. $N$ Engl J Med. 2000;343(22):1594-1602.
73. Hanauer SB, et al. Maintenance infliximab for Crohn's disease: the ACCENT I randomised trial. Lancet. 2002;359(9317):1541-1549.

74. Braun J, et al. Treatment of active ankylosing spondylitis with infliximab: a randomised controlled multicentre trial. Lancet. 2002;359(9313):1187-1193

75. Palladino MA, Bahjat FR, Theodorakis EA, Moldawer LL. Anti-TNF-alpha therapies: the next generation. Nat Rev Drug Discov. 2003;2(9):736-746.

76. Ruffell D, et al. A CREB-C/EBPbeta cascade induces M2 macrophage-specific gene expression and promotes muscle injury repair. Proc Natl Acad Sci US A. 2009;106(41):17475-17480.

77. Nahrendorf $M$, et al. The healing myocardium sequentially mobilizes two monocyte subsets with divergent and complementary functions. J Exp Med. 2007;204(12):3037-3047.

78. Arnold L, et al. Inflammatory monocytes recruited after skeletal muscle injury switch into antiinflammatory macrophages to support myogenesis. J Exp Med. 2007;204(5):1057-1069.

79. Zamboni P. The big idea: iron-dependent inflammation in venous disease and proposed parallels in multiple sclerosis. J R Soc Med. 2006;99(11):589-593.

80. Stadler N, Lindner RA, Davies MJ. Direct detection and quantification of transition metal ions in human atherosclerotic plaques: evidence for the presence of elevated levels of iron and copper. Arterioscler Thromb Vasc Biol. 2004;24(5):949-954.

81. Mendez MV, Stanley A, Park HY, Shon K, Phillips T, Menzoian JO. Fibroblasts cultured from venous ulcers display cellular characteristics of senescence. J Vasc Surg. 1998;28(5):876-883.

82. Yuan XM, Li W. The iron hypothesis of atherosclerosis and its clinical impact. Ann Med. 2003;35(8):578-591.

83. Eklof B, et al. Revision of the CEAP classification for chronic venous disorders: consensus statement. J Vasc Surg. 2004;40(6):1248-1252. 